\title{
Interval Oscillation Criteria for Second-Order Nonlinear Forced Dynamic Equations with Damping on Time Scales
}

\author{
Yibing Sun, Zhenlai Han, Shurong Sun, and Chao Zhang \\ School of Mathematical Sciences, University of Jinan, Jinan, Shandong 250022, China \\ Correspondence should be addressed to Zhenlai Han; hanzhenlai@163.com
}

Received 27 November 2012; Revised 28 January 2013; Accepted 31 January 2013

Academic Editor: Patricia J. Y. Wong

Copyright (C) 2013 Yibing Sun et al. This is an open access article distributed under the Creative Commons Attribution License, which permits unrestricted use, distribution, and reproduction in any medium, provided the original work is properly cited.

\begin{abstract}
By using the Riccati transformation technique and constructing a class of Philos-type functions on time scales, we establish some new interval oscillation criteria for the second-order damped nonlinear dynamic equations with forced term of the form $\left(r(t) x^{\Delta}(t)\right)^{\Delta}+p(t) x^{\Delta \sigma}(t)+q(t)\left(x^{\sigma}(t)\right)^{\alpha}=F\left(t, x^{\sigma}(t)\right)$ on a time scale $\mathbb{T}$ which is unbounded, where $\alpha$ is a quotient of odd positive integer. Our results in this paper extend and improve some known results. Some examples are given here to illustrate our main results.
\end{abstract}

\section{Introduction}

In this paper, we are concerned with the oscillation criteria for the following forced second-order nonlinear dynamic equations with damping:

$$
\left(r(t) x^{\Delta}(t)\right)^{\Delta}+p(t) x^{\Delta \sigma}(t)+q(t)\left(x^{\sigma}(t)\right)^{\alpha}=F\left(t, x^{\sigma}(t)\right)
$$

on a time scale $\mathbb{T}$, where $\alpha$ is a quotient of odd positive integer. Throughout this paper and without further mention, we assume that the functions $r, p, q \in C_{\mathrm{rd}}\left(\left[t_{0}, \infty\right)_{\mathbb{T}}, \mathbb{R}\right), F \in$ $C(\mathbb{T} \times \mathbb{R}, \mathbb{R})$ with $r(t)>0, p(t) \leq 0$, and $p / r^{\sigma} \in \mathscr{R}^{+}$.

The theory of time scales, which has recently received a lot of attention, was originally introduced by Stefan Hilger in his Ph.D. thesis in 1988 (see [1]). Since then a rapidly expanding body of the literature has sought to unify, extend, and generalize ideas from discrete calculus, quantum calculus, and continuous calculus to arbitrary time scale calculus, where a time scale is an arbitrary nonempty closed subset of the real numbers $\mathbb{R}$, and the cases when this time scale is equal to the reals or to the integers represent the classical theories of differential and of difference equations. Many other interesting time scales exist, and they give rise to many applications (see [2]). Not only does the new theory of the so-called dynamic equations unify the theories of differential equations and difference equations, but also it extends these classical cases to cases "in between", for example, to the socalled $q$-difference equations when $\mathbb{T}=q^{\mathbb{N}_{0}}=\left\{q^{t}: t \in\right.$ $\left.\mathbb{N}_{0}, q>1\right\}$ (which has important applications in quantum theory) and can be applied on different types of time scales like $\mathbb{T}=h \mathbb{N}, \mathbb{T}=\mathbb{N}^{2}$, and $\mathbb{T}=\mathbb{T}_{n}$, the space of the harmonic numbers. A book on the subject of time scales by Bohner and Peterson [2] summarizes and organizes much of the time scale calculus. For advances of dynamic equations on the time scales we refer the reader to the book [3].

Since we are interested in the oscillatory behavior of solutions near infinity, we make the assumption throughout this paper that the given time scale $\mathbb{T}$ is unbounded above. We assume $t_{0} \in \mathbb{T}$ and it is convenient to assume $t_{0}>0$. We define the time scale interval of the form $\left[t_{0}, \infty\right)_{\mathbb{T}}$ by $\left[t_{0}, \infty\right)_{\mathbb{T}}=\left[t_{0}, \infty\right) \cap \mathbb{T}$. We assume throughout that $\mathbb{T}$ has the topology that it inherits from the standard topology on the real numbers $\mathbb{R}$.

By a solution of (1), we mean a nontrivial real-valued function $x$ satisfying $(1)$ on $\left[t_{x}, \infty\right)_{\mathbb{T}}$. A solution $x$ of (1) is said to be oscillatory on $\left[t_{x}, \infty\right)_{\mathbb{T}}$ in case it is neither eventually positive nor eventually negative; otherwise, it is 
nonoscillatory. Equation (1) is said to be oscillatory in case all its solutions are oscillatory. Our attention is restricted to those solutions of (1) which exist on some half line $\left[t_{x}, \infty\right)_{\mathbb{T}}$ and satisfy $\sup \{|x(t)|: t \geq T\}>0$ for all $T \geq t_{x}$.

In recent years, there has been much research activity concerning the interval oscillation criteria for various second order differential equations; see [4-9]. A great deal of effort has been spent in obtaining criteria for oscillation of dynamic equations on time scales without forcing terms and it is usually assumed that the potential function $q$ is positive. We refer the reader to the papers [10-25] and the references cited therein. On the other hand, there has been an increasing interest in obtaining sufficient conditions for the oscillation and nonoscillation of solutions of dynamic equations with forcing terms on time scales, and we refer the reader to the papers [26-35].

In 2004, by using two inequalities due to Hölder and Hardy and Littlewood and Polya as well as averaging functions, Li [4] established several interval oscillation criteria for the second order damped quasilinear differential equation with forced term of the following form:

$$
\begin{aligned}
& \left(r(t)\left|y^{\prime}(t)\right|^{\alpha-1} y^{\prime}(t)\right)^{\prime}+p(t)\left|y^{\prime}(t)\right|^{\alpha-1} y^{\prime}(t) \\
& +q(t)|y(t)|^{\beta-1} y(t)=e(t)
\end{aligned}
$$

where $r \in C^{1}\left(\left[t_{0}, \infty\right), \mathbb{R}^{+}\right)$, and $\beta>\alpha>0$ are constants. The obtained results were based on the information only on a sequence of subintervals of $\left[t_{0}, \infty\right)$, rather than on the whole half line, made use of the oscillatory properties of the forcing term, and extended a known result which is obtained by means of a Picone identity.

Erbe et al. [26] studied the forced second-order nonlinear dynamic equation

$$
\left(p(t) x^{\Delta}(t)\right)^{\Delta}+q(t)\left|x^{\sigma}(t)\right|^{\gamma} \operatorname{sgn} x^{\sigma}(t)=f(t)
$$

on a time scale $\mathbb{T}$, where $\gamma \geq 1$. By using the Riccati substitution, the authors established some new interval oscillation criteria, that is, the criteria given by the behavior of $q$ and $f$ on a sequence of subintervals of $[a, \infty)_{\mathbb{T}}$.

In [31], by constructing a class of Philos-type functions on time scales, Li et al. established some oscillation criteria for the second order nonlinear dynamic equations with the forced term

$$
x^{\Delta \Delta}(t)+a(t) f(x(q(t)))=e(t)
$$

on a time scale $\mathbb{T}$, where $a, q$, and $e$ are real-valued rdcontinuous functions defined on $\mathbb{T}$, with $q: T \rightarrow T, q(t) \rightarrow$ $\infty$ as $t \rightarrow \infty$, and $f \rightarrow C(\mathbb{R}, \mathbb{R}), x f(x)>0$ whenever $x \neq 0$. The obtained results unified the oscillation of the second order forced differential equation and the second order forced difference equation. An example was considered to illustrate the main results in the end.
Erbe et al. [32] were concerned with the oscillatory behavior of the forced second-order functional dynamic equation with mixed nonlinearities

$$
\left(a(t) x^{\Delta}(t)\right)^{\Delta}+\sum_{i=0}^{n} p_{i}(t)\left|x\left(\tau_{i}(t)\right)\right|^{\alpha_{i}} \operatorname{sgn} x\left(\tau_{i}(t)\right)=e(t)
$$

on an arbitrary time scale $\mathbb{T}$, where $\alpha_{0}=1, \alpha_{1}>\alpha_{2}>$ $\cdots>\alpha_{m}>1>\alpha_{m+1}>\cdots>\alpha_{n}$, and $\tau_{i}: \mathbb{T} \rightarrow \mathbb{\mathbb { T }}$ are nondecreasing rd-continuous functions on $\mathbb{R}, \tau_{i}(t) \leq \sigma(t)$, and $\lim _{t \rightarrow \infty} \tau_{i}(t)=\infty$, for $i=0,1, \ldots, n$. Their results in a particular case solved a problem posed by Anderson, and their results in the special cases when the time scale is the set of real numbers and the set of integers involved and improved some oscillation results for second-order differential and difference equations, respectively.

In this paper, we intend to use the Riccati transformation technique to obtain some interval oscillation criteria for (1). Our results do not require that $q$ and $f$ be of definite sign and are based on the information only on a sequence of subintervals of $\left[t_{0}, \infty\right]_{\mathbb{T}}$ rather than the whole half line. To the best of our knowledge, nothing is known regarding the oscillation behavior of (1) on time scales until now, and there are few results regarding the interval oscillation criteria for (1) on time scales without the damping term when $\alpha<1$, so our results expand the known scope of the study.

The paper is organized as follows. In Section 2, we present some basic definitions and useful results from the theory of calculus on time scales on which we rely in the later section. In Section 3, we intend to use the Riccati transformation technique, integral averaging technique, and inequalities to obtain some sufficient conditions for oscillation of every solution of (1). In Section 4, we give two examples to illustrate Theorems 3 and 7, respectively.

\section{Some Preliminaries}

On any time scale $\mathbb{T}$, we define the forward and the backward jump operators by

$$
\begin{aligned}
& \sigma(t)=\inf \{s \in \mathbb{T}: s>t\}, \\
& \rho(t)=\sup \{s \in \mathbb{T}: s<t\},
\end{aligned}
$$

where inf $\emptyset=\sup \mathbb{T}$ and $\sup \emptyset=\inf \mathbb{T}$. A point $t \in \mathbb{T}$ is said to be left-dense if $\rho(t)=t$, right-dense if $\sigma(t)=t$, left-scattered if $\rho(t)<t$, and right-scattered if $\sigma(t)>t$. The graininess function $\mu$ for a time scale $\mathbb{T}$ is defined by $\mu(t)=\sigma(t)-t$.

For a function $f: \mathbb{T} \rightarrow \mathbb{R}$, the (delta) derivative is defined by

$$
f^{\Delta}(t)=\frac{f(\sigma(t))-f(t)}{\sigma(t)-t}
$$

if $f$ is continuous at $t$ and $t$ is right-scattered. If $t$ is rightdense, then the derivative is defined by

$$
f^{\Delta}(t)=\lim _{s \longrightarrow t^{+}} \frac{f(\sigma(t))-f(s)}{\sigma(t)-s}=\lim _{s \longrightarrow t^{+}} \frac{f(t)-f(s)}{t-s},
$$


provided this limit exists. A function $f: \mathbb{T} \rightarrow \mathbb{R}$ is said to be rd-continuous provided $f$ is continuous at right-dense points and there exists a finite left limit at all left-dense points in $\mathbb{T}$. The set of all such rd-continuous functions is denoted by $C_{\text {rd }}(\mathbb{T})$. The derivative $f^{\Delta}$ of $f$ and the forward jump operator $\sigma$ are related by the formula

$$
f^{\sigma}(t)=f(\sigma(t))=f(t)+\mu(t) f^{\Delta}(t) .
$$

Also, we will use $x^{\Delta \sigma}$ which is shorthand for $\left(x^{\Delta}\right)^{\sigma}$ to denote $x^{\Delta}(t)+\mu(t) x^{\Delta \Delta}(t)$. We will make use of the following product and quotient rules for the derivative of two differentiable functions $f$ and $g$ :

$$
\begin{gathered}
(f g)^{\Delta}(t)=f^{\Delta}(t) g(t)+f^{\sigma}(t) g^{\Delta}(t) \\
=f(t) g^{\Delta}(t)+f^{\Delta}(t) g^{\sigma}(t), \\
\left(\frac{f}{g}\right)^{\Delta}(t)=\frac{f^{\Delta}(t) g(t)-f(t) g^{\Delta}(t)}{g(t) g^{\sigma}(t)}, \quad \text { if } g g^{\sigma} \neq 0 .
\end{gathered}
$$

The integration by parts formula reads

$$
\begin{aligned}
\int_{b}^{c} f^{\Delta}(t) g(t) \Delta t= & f(c) g(c)-f(b) g(b) \\
& -\int_{b}^{c} f^{\sigma}(t) g^{\Delta}(t) \Delta t .
\end{aligned}
$$

We say that a function $p: \mathbb{T} \rightarrow \mathbb{R}$ is regressive provided

$$
1+\mu(t) p(t) \neq 0, \quad \forall t \in \mathbb{T} .
$$

The set of all regressive and rd-continuous functions $f: \mathbb{T} \rightarrow$ $\mathbb{R}$ will be denoted by

$$
\mathscr{R}=\mathscr{R}(\mathbb{\mathbb { T }})=\mathscr{R}(\mathbb{T}, \mathbb{R}) .
$$

If $p \in \mathscr{R}$, then we can define the exponential function by

$$
e_{p}(t, s)=\exp \left(\int_{s}^{t} \xi_{\mu(\tau)}(p(\tau)) \Delta \tau\right) \quad \text { for } s, t \in \mathbb{T},
$$

where $\xi_{h}(z)$ is the cylinder transformation, which is defined by

$$
\xi_{h}(z)= \begin{cases}\frac{\log (1+h z)}{h}, & h \neq 0, \\ z, & h=0 .\end{cases}
$$

Next, we give the following lemmas which will be used in the proof of our main results.

Lemma 1 (see [2, Chapter 2]). If $g \in \mathscr{R}^{+}$; that is, $g: \mathbb{T} \rightarrow \mathbb{R}$ is rd-continuous and such that $1+\mu(t) g(t)>0$ for all $t \in$ $\left[t_{0}, \infty\right)_{\mathbb{T}}$, then the initial value problem $y^{\Delta}=g(t) y, y\left(t_{0}\right)=$ $y_{0} \in \mathbb{R}$ has a unique and positive solution on $\left[t_{0}, \infty\right)_{\mathbb{T}}$, denoted by $e_{g}\left(t, t_{0}\right) y_{0}$. This "exponential function" $e_{g}\left(\cdot, t_{0}\right)$ satisfies the semigroup property $e_{g}(a, b) e_{g}(b, c)=e_{g}(a, c)$.

Lemma 2 (see [36]). If $\lambda>1$ and $\rho>1$ are conjugate numbers $(1 / \lambda+1 / \rho=1)$, then for any $X, Y \in \mathbb{R}$,

$$
\frac{|X|^{\lambda}}{\lambda}+\frac{|Y|^{\rho}}{\rho} \geq|X Y| \text {. }
$$

\section{Main Results}

Now, we are in a position to state and prove some new results which guarantee that every solution of (1) oscillates. In the sequel, we say that a function $u$ belongs to a function class

$$
\xi(a, b):=\left\{u \in C_{\mathrm{rd}}^{1}[a, b]_{\mathbb{T}}: u(a)=u(b)=0, u(t) \not \equiv 0\right\},
$$

denoted by $u \in \xi(a, b)$.

Theorem 3. Assume that $\alpha>1$ and for any $T \in\left[t_{0}, \infty\right)_{\mathbb{T}}$, there exist constants $a_{k}$ and $b_{k} \in[T, \infty)_{\mathbb{T}}$, such that $a_{k}<b_{k}$, $k=1,2$, with

$$
\begin{gathered}
q(t) \geq 0, \quad \text { for } t \in\left[a_{1}, b_{1}\right]_{\mathbb{T}} \cup\left[a_{2}, b_{2}\right]_{\mathbb{T}}, \\
(-1)^{k} F\left(t, x^{\sigma}(t)\right) \geq(-1)^{k} f(t) \geq 0, \quad \text { for } t \in\left[a_{k}, b_{k}\right]_{\mathbb{T}}, \\
k=1,2,
\end{gathered}
$$

where $f \in C_{r d}\left(\left[t_{0}, \infty\right)_{\mathbb{T}}, \mathbb{R}\right)$. Furthermore, assume that there exist functions $\eta \in C_{r d}^{1}\left(\left[t_{0}, \infty\right)_{\mathbb{T}}, \mathbb{R}^{+}\right), \eta^{\Delta}(t) \geq 0$, and $u \in$ $\xi\left(a_{k}, b_{k}\right), k=1,2$, such that

$$
\begin{array}{r}
\int_{a_{k}}^{b_{k}}\left(\eta(t) r(t)\left(u^{\Delta}(t)\right)^{2}-P\left(t, a_{k}\right)\left(u^{\sigma}(t)\right)^{2}\right) \Delta t \leq 0, \\
k=1,2,
\end{array}
$$

where

$$
\begin{gathered}
P\left(t, a_{k}\right)=\delta_{0}(t)-\eta^{\Delta}(t) \delta_{1}\left(t, a_{k}\right) \\
+\frac{\eta^{\sigma}(t) p(t)}{r^{\sigma}(t)} \delta_{1}\left(\sigma(t), a_{k}\right), \\
\delta_{0}(t)=\alpha^{1 / \alpha}\left(\frac{\alpha}{\alpha-1}\right)^{(\alpha-1) / \alpha} \eta^{\sigma}(t) q^{1 / \alpha}(t)|f(t)|^{(\alpha-1) / \alpha}, \\
\delta_{1}\left(t, a_{k}\right)= \\
e_{p / r^{\sigma}}\left(t, a_{k}\right) \\
\times\left(\int_{a_{k}}^{t} \frac{1}{r(s) e_{p / r^{\sigma}}\left(s, a_{k}\right)} \Delta s\right)^{-1} .
\end{gathered}
$$

Then (1) is oscillatory on $\left[t_{0}, \infty\right)_{\mathbb{T}}$.

Proof. Assume that $x$ is a nonoscillatory solution of (1) on $\left[t_{0}, \infty\right)_{\mathbb{T}}$. Without loss of generality, we may assume that there exists a $t_{1} \in\left[t_{0}, \infty\right)_{\mathbb{T}}$, such that $x(t)>0, x^{\sigma}(t)>0$ for all $t \in\left[t_{1}, \infty\right)_{\mathbb{T}}$. By assumption, we can choose $b_{1}>a_{1}>t_{1}$, then $q(t) \geq 0$ and $F\left(t, x^{\sigma}(t)\right) \leq 0$ on the interval $\left[a_{1}, b_{1}\right]_{\mathbb{T}}$. From (1), we have

$$
\left(r(t) x^{\Delta}(t)\right)^{\Delta}+p(t) x^{\Delta \sigma}(t) \leq 0 .
$$

Using Lemma 1 and the above inequality, we get

$$
\left(r(t) x^{\Delta}(t) e_{p / r^{\sigma}}\left(t, a_{1}\right)\right)^{\Delta} \leq 0 .
$$


Hence $r(t) x^{\Delta}(t) e_{p / r^{\sigma}}\left(t, a_{1}\right)$ is nonincreasing on $\left[a_{1}, b_{1}\right]_{\mathbb{T}}$. So for $t \in\left[a_{1}, b_{1}\right]_{\mathbb{T}}$,

$$
\begin{aligned}
x(t) & >x(t)-x\left(a_{1}\right)=\int_{a_{1}}^{t} \frac{r(s) x^{\Delta}(s) e_{p / r^{\sigma}}\left(s, a_{1}\right)}{r(s) e_{p / r^{\sigma}}\left(s, a_{1}\right)} \Delta s \\
& \geq r(t) x^{\Delta}(t) e_{p / r^{\sigma}}\left(t, a_{1}\right) \int_{a_{1}}^{t} \frac{1}{r(s) e_{p / r^{\sigma}}\left(s, a_{1}\right)} \Delta s .
\end{aligned}
$$

Therefore,

$$
\begin{aligned}
\frac{r(t) x^{\Delta}(t)}{x(t)} & <\frac{1}{e_{p / r^{\sigma}}\left(t, a_{1}\right)}\left(\int_{a_{1}}^{t} \frac{1}{r(s) e_{p / r^{\sigma}}\left(s, a_{1}\right)} \Delta s\right)^{-1} \\
& =\delta_{1}\left(t, a_{1}\right) .
\end{aligned}
$$

Define the function $\omega$ by

$$
\omega(t)=\eta(t) \frac{r(t) x^{\Delta}(t)}{x(t)}, \quad t \in\left[a_{1}, b_{1}\right]_{\mathbb{T}} .
$$

Using the product rule and the quotient rule, we obtain

$$
\begin{aligned}
\omega^{\Delta}(t)= & \eta^{\sigma}(t) \frac{\left(r(t) x^{\Delta}(t)\right)^{\Delta} x(t)-r(t)\left(x^{\Delta}(t)\right)^{2}}{x(t) x^{\sigma}(t)} \\
& +\eta^{\Delta}(t) \frac{r(t) x^{\Delta}(t)}{x(t)} .
\end{aligned}
$$

In view of (1), (26), and (27), we have

$$
\begin{aligned}
\omega^{\Delta}(t)= & -\eta^{\sigma}(t) \frac{p(t) x^{\Delta \sigma}(t)}{x^{\sigma}(t)}-\eta^{\sigma}(t) q(t)\left(x^{\sigma}(t)\right)^{\alpha-1} \\
& +\eta^{\sigma}(t) \frac{F\left(t, x^{\sigma}(t)\right)}{x^{\sigma}(t)}-\eta^{\sigma}(t) \frac{r(t)\left(x^{\Delta}(t)\right)^{2}}{x(t) x^{\sigma}(t)} \\
& +\eta^{\Delta}(t) \frac{r(t) x^{\Delta}(t)}{x(t)} \\
= & -\frac{\eta^{\sigma}(t) p(t) \frac{r^{\sigma}(t) x^{\Delta \sigma}(t)}{r^{\sigma}(t)}-\eta^{\sigma}(t) q(t)\left(x^{\sigma}(t)\right)^{\alpha-1}}{} \\
& -\eta^{\sigma}(t) \frac{\left|F\left(t, x^{\sigma}(t)\right)\right|}{x^{\sigma}(t)}-\frac{\eta^{\sigma}(t) x(t)}{\eta^{2}(t) r(t) x^{\sigma}(t)} \omega^{2}(t) \\
& +\eta^{\Delta}(t) \frac{r(t) x^{\Delta}(t)}{x(t)} .
\end{aligned}
$$

From (19), (25), and (28), we get

$$
\begin{aligned}
\omega^{\Delta}(t) \leq & -\frac{\eta^{\sigma}(t) p(t)}{r^{\sigma}(t)} \delta_{1}\left(\sigma(t), a_{1}\right)-\eta^{\sigma}(t) q(t)\left(x^{\sigma}(t)\right)^{\alpha-1} \\
& -\eta^{\sigma}(t) \frac{|f(t)|}{x^{\sigma}(t)}-\frac{\eta^{\sigma}(t) x(t)}{\eta^{2}(t) r(t) x^{\sigma}(t)} \omega^{2}(t) \\
& +\eta^{\Delta}(t) \delta_{1}\left(t, a_{1}\right) .
\end{aligned}
$$

Set

$$
\begin{gathered}
G(x)=\eta^{\sigma}(t) q(t) x^{\alpha-1}+\eta^{\sigma}(t) \frac{|f(t)|}{x}, \\
\lambda=\alpha, \quad \rho=\frac{\alpha}{\alpha-1} .
\end{gathered}
$$

From Lemma 2, it is easy to see that

$$
\begin{aligned}
G\left(x^{\sigma}\right) & \geq \alpha^{1 / \alpha}\left(\frac{\alpha}{\alpha-1}\right)^{(\alpha-1) / \alpha} \eta^{\sigma}(t) q^{1 / \alpha}(t)|f(t)|^{(\alpha-1) / \alpha} \\
& =\delta_{0}(t) .
\end{aligned}
$$

Since $x(t)>0$, we obtain

$$
\begin{aligned}
0<\frac{x(t)}{r(t) x^{\sigma}(t)} & =\frac{1}{r(t)+\mu(t)\left(r(t) x^{\Delta}(t) / x(t)\right)} \\
& =\frac{\eta(t)}{\eta(t) r(t)+\mu(t) \omega(t)} .
\end{aligned}
$$

Thus, combining (29)-(32) and noticing that $\eta^{\Delta}(t) \geq 0$, we have

$$
\omega^{\Delta}(t) \leq-P\left(t, a_{1}\right)-\frac{1}{\eta(t) r(t)+\mu(t) \omega(t)} \omega^{2}(t),
$$

where $P$ is defined as in Theorem 3. Multiplying (33) by $\left(u^{\sigma}(t)\right)^{2}$ and integrating from $a_{1}$ to $b_{1}$, we get

$$
\begin{aligned}
\int_{a_{1}}^{b_{1}}\left(u^{\sigma}(t)\right)^{2} \omega^{\Delta}(t) \Delta t \leq & -\int_{a_{1}}^{b_{1}} P\left(t, a_{1}\right)\left(u^{\sigma}(t)\right)^{2} \Delta t \\
& -\int_{a_{1}}^{b_{1}} \frac{\left(u^{\sigma}(t)\right)^{2} \omega^{2}(t)}{\eta(t) r(t)+\mu(t) \omega(t)} \Delta t
\end{aligned}
$$

Using integration by parts on the first integral, we obtain

$$
\begin{aligned}
& \left.u^{2}(t) \omega(t)\right|_{a_{1}} ^{b_{1}}-\int_{a_{1}}^{b_{1}}\left(u(t)+u^{\sigma}(t)\right) u^{\Delta}(t) \omega(t) \Delta t \\
& \leq-\int_{a_{1}}^{b_{1}} P\left(t, a_{1}\right)\left(u^{\sigma}(t)\right)^{2} \Delta t-\int_{a_{1}}^{b_{1}} \frac{\left(u^{\sigma}(t)\right)^{2} \omega^{2}(t)}{\eta(t) r(t)+\mu(t) \omega(t)} \Delta t .
\end{aligned}
$$


Rearranging and using $u\left(a_{1}\right)=0=u\left(b_{1}\right)$, we have

$$
\begin{gathered}
0 \geq \int_{a_{1}}^{b_{1}} \frac{\left(u^{\sigma}(t)\right)^{2} \omega^{2}(t)}{\eta(t) r(t)+\mu(t) \omega(t)} \Delta t \\
\quad-\int_{a_{1}}^{b_{1}}\left(u(t)+u^{\sigma}(t)\right) u^{\Delta}(t) \omega(t) \Delta t \\
\quad+\int_{a_{1}}^{b_{1}} P\left(t, a_{1}\right)\left(u^{\sigma}(t)\right)^{2} \Delta t \\
=\int_{a_{1}}^{b_{1}} \frac{\left(u^{\sigma}(t)\right)^{2} \omega^{2}(t)}{\eta(t) r(t)+\mu(t) \omega(t)} \Delta t \\
\quad-\int_{a_{1}}^{b_{1}}\left(2 u^{\sigma}(t) u^{\Delta}(t) \omega(t)\right. \\
+\int_{a_{1}}^{b_{1}} P\left(t, a_{1}\right)\left(u^{\sigma}(t)\right)^{2} \Delta t .
\end{gathered}
$$

Adding and subtracting the term $\int_{a_{1}}^{b_{1}} \eta(t) r(t)\left(u^{\Delta}(t)\right)^{2} \Delta t$ and using (20), we get

$$
\begin{aligned}
& 0 \geq \int_{a_{1}}^{b_{1}}\left[\frac{\left(u^{\sigma}(t)\right)^{2} \omega^{2}(t)}{\eta(t) r(t)+\mu(t) \omega(t)}-2 u^{\sigma}(t) u^{\Delta}(t) \omega(t)\right. \\
& \left.+(\eta(t) r(t)+\mu(t) \omega(t))\left(u^{\Delta}(t)\right)^{2}\right] \Delta t \\
& -\int_{a_{1}}^{b_{1}}\left(\eta(t) r(t)\left(u^{\Delta}(t)\right)^{2}-P\left(t, a_{1}\right)\left(u^{\sigma}(t)\right)^{2}\right) \Delta t \\
& \geq \int_{a_{1}}^{b_{1}}\left[\frac{u^{\sigma}(t) \omega(t)}{\sqrt{\eta(t) r(t)+\mu(t) \omega(t)}}\right. \\
& \left.-\sqrt{\eta(t) r(t)+\mu(t) \omega(t)} u^{\Delta}(t)\right]^{2} \Delta t .
\end{aligned}
$$

It follows that

$$
\begin{aligned}
\int_{a_{1}}^{b_{1}} & \frac{u^{\sigma}(t) \omega(t)}{\sqrt{\eta(t) r(t)+\mu(t) \omega(t)}} \\
& \left.-\sqrt{\eta(t) r(t)+\mu(t) \omega(t)} u^{\Delta}(t)\right]^{2} \Delta t=0 .
\end{aligned}
$$

This implies that

$$
\begin{aligned}
& \frac{u^{\sigma}(t) \omega(t)}{\sqrt{\eta(t) r(t)+\mu(t) \omega(t)}} \\
& -\sqrt{\eta(t) r(t)+\mu(t) \omega(t)} u^{\Delta}(t)=0, \quad t \in\left[a_{1}, b_{1}\right]_{\mathbb{T}} .
\end{aligned}
$$

Solving for $u^{\Delta}$, we get that $u$ solves the IVP

$$
\begin{aligned}
u^{\Delta}(t)= & \frac{\omega(t)}{\eta(t) r(t)+\mu(t) \omega(t)} u^{\sigma}(t), \\
& u\left(a_{1}\right)=0, \quad \text { for } t \in\left[a_{1}, b_{1}\right]_{\mathbb{T}} .
\end{aligned}
$$

Since $-\omega /(\eta r+\mu \omega) \in \mathscr{R}$, we obtain from [2, Theorem 2.7.1] that $u(t) \equiv 0$ on $\left[a_{1}, b_{1}\right]_{\mathbb{T}}$, which is a contradiction. The proof when $x$ is eventually negative follows the same arguments using the interval $\left[a_{2}, b_{2}\right]_{\mathbb{V}}$ instead of $\left[a_{1}, b_{1}\right]_{\mathbb{T}}$, where we use $q(t) \geq 0, F\left(t, x^{\sigma}(t)\right) \geq 0$ on $\left[a_{2}, b_{2}\right]_{\mathbb{V}}$, and $\int_{a_{2}}^{b_{2}}\left(\eta(t) r(t)\left(u^{\Delta}(t)\right)^{2}-\right.$ $\left.P(t)\left(u^{\sigma}(t)\right)^{2}\right) \Delta t \leq 0$. The proof is complete.

Remark 4. When $p(t)=0$ and $F\left(t, x^{\sigma}(t)\right)=f(t)$, Theorem 3 contains Theorem 3.2 in [26].

Theorem 5. Assume that $\alpha=1$ and for any $T \in\left[t_{0}, \infty\right)_{\mathbb{T}}$, there exist constants $a_{k}$ and $b_{k} \in[T, \infty)_{\mathbb{T}}$, such that $a_{k}<b_{k}$, $k=1,2$, with

$$
\begin{gathered}
q(t) \geq 0, \quad \text { for } t \in\left[a_{1}, b_{1}\right]_{\mathbb{V}} \cup\left[a_{2}, b_{2}\right]_{\mathbb{T}}, \\
(-1)^{k} F\left(t, x^{\sigma}(t)\right) \geq 0, \quad \text { for } t \in\left[a_{k}, b_{k}\right]_{\mathbb{T}}, k=1,2 .
\end{gathered}
$$

Furthermore, assume that there exist functions $\eta \in C_{r d}^{1}\left(\left[t_{0}, \infty\right)_{\mathbb{T}}, \mathbb{R}^{+}\right), \eta^{\Delta}(t) \geq 0$, and $u \in \xi\left(a_{k}, b_{k}\right)$, $k=1,2$, such that

$$
\begin{array}{r}
\int_{a_{k}}^{b_{k}}\left(\eta(t) r(t)\left(u^{\Delta}(t)\right)^{2}-K\left(t, a_{k}\right)\left(u^{\sigma}(t)\right)^{2}\right) \Delta t \leq 0, \\
k=1,2,
\end{array}
$$

where

$$
\begin{aligned}
K\left(t, a_{k}\right)= & \eta^{\sigma}(t) q(t)-\eta^{\Delta}(t) \delta_{1}\left(t, a_{k}\right) \\
& +\frac{\eta^{\sigma}(t) p(t)}{r^{\sigma}(t)} \delta_{1}\left(\sigma(t), a_{k}\right),
\end{aligned}
$$

and $\delta_{1}$ is defined as in Theorem 3. Then (1) is oscillatory on $\left[t_{0}, \infty\right)_{\mathbb{T}}$.

Proof. Assume that $x$ is a nonoscillatory solution of (1) on $\left[t_{0}, \infty\right)_{\mathbb{T}}$. Without loss of generality, we may assume that there exists a $t_{1} \in\left[t_{0}, \infty\right)_{\mathbb{T}}$, such that $x(t)>0, x^{\sigma}(t)>0$ for all $t \in\left[t_{1}, \infty\right)_{\mathbb{T}}$. By assumption, we can choose $b_{1}>a_{1}>t_{1}$, then $q(t) \geq 0$ and $F\left(t, x^{\sigma}(t)\right) \leq 0$ on the interval $\left[a_{1}, b_{1}\right]_{\mathbb{T}}$. We define $\omega$ as in Theorem 3. Proceeding as in the proof of Theorem 3 and from (25) and (32), we get

$$
\begin{aligned}
\omega^{\Delta}(t)= & -\frac{\eta^{\sigma}(t) p(t)}{r^{\sigma}(t)} \frac{r^{\sigma}(t) x^{\Delta \sigma}(t)}{x^{\sigma}(t)}-\eta^{\sigma}(t) q(t) \\
& -\eta^{\sigma}(t) \frac{\left|F\left(t, x^{\sigma}(t)\right)\right|}{x^{\sigma}(t)} \\
& -\frac{\eta^{\sigma}(t) x(t)}{\eta^{2}(t) r(t) x^{\sigma}(t)} \omega^{2}(t)+\eta^{\Delta}(t) \frac{r(t) x^{\Delta}(t)}{x(t)} \\
\leq & -K\left(t, a_{1}\right)-\frac{1}{\eta(t) r(t)+\mu(t) \omega(t)} \omega^{2}(t),
\end{aligned}
$$


where $K$ is defined as in Theorem 5. Multiplying (44) by $\left(u^{\sigma}(t)\right)^{2}$ and integrating from $a_{1}$ to $b_{1}$, we get

$$
\begin{aligned}
\int_{a_{1}}^{b_{1}}\left(u^{\sigma}(t)\right)^{2} \omega^{\Delta}(t) \Delta t \leq & -\int_{a_{1}}^{b_{1}} K\left(t, a_{1}\right)\left(u^{\sigma}(t)\right)^{2} \Delta t \\
& -\int_{a_{1}}^{b_{1}} \frac{\left(u^{\sigma}(t)\right)^{2} \omega^{2}(t)}{\eta(t) r(t)+\mu(t) \omega(t)} \Delta t .
\end{aligned}
$$

The rest of the argument proceeds as in Theorem 3 to get a contradiction to (42). The proof is complete.

Remark 6. When $p(t)=0$ and $F\left(t, x^{\sigma}(t)\right)=f(t)$, Theorem 5 contains Theorem 2.1 in [26].

Theorem 7. Assume that $\alpha<1$ and for any $T \in\left[t_{0}, \infty\right)_{\mathbb{T}}$, there exist constants $a_{k}$ and $b_{k} \in[T, \infty)_{\mathbb{T}}$, such that $a_{k}<b_{k}$, $k=1,2$, with

$$
\begin{array}{r}
q(t) \geq 0, \quad \text { for } t \in\left[a_{1}, b_{1}\right]_{\mathbb{T}} \cup\left[a_{2}, b_{2}\right]_{\mathbb{T}}, \\
(-1)^{k} F\left(t, x^{\sigma}(t)\right) \geq(-1)^{k} f(t)\left(x^{\sigma}(t)\right)^{\alpha+1} \geq 0 \\
\text { for } t \in\left[a_{k}, b_{k}\right]_{\mathbb{T}}, \quad k=1,2,
\end{array}
$$

where $f \in C_{r d}\left(\left[t_{0}, \infty\right)_{\mathbb{T}}, \mathbb{R}\right)$. Furthermore, assume that there exist functions $\eta \in C_{r d}^{1}\left(\left[t_{0}, \infty\right)_{\mathbb{T}}, \mathbb{R}^{+}\right), \eta^{\Delta}(t) \geq 0$, and $u \in$ $\xi\left(a_{k}, b_{k}\right), k=1,2$, such that

$$
\begin{array}{r}
\int_{a_{k}}^{b_{k}}\left(\eta(t) r(t)\left(u^{\Delta}(t)\right)^{2}-P_{1}\left(t, a_{k}\right)\left(u^{\sigma}(t)\right)^{2}\right) \Delta t \leq 0, \\
k=1,2,
\end{array}
$$

where

$$
\begin{gathered}
P_{1}\left(t, a_{k}\right)=\delta_{2}(t)-\eta^{\Delta}(t) \delta_{1}\left(t, a_{k}\right) \\
+\frac{\eta^{\sigma}(t) p(t)}{r^{\sigma}(t)} \delta_{1}\left(\sigma(t), a_{k}\right), \\
\delta_{2}(t)=\frac{1}{\alpha^{\alpha}(1-\alpha)^{1-\alpha}} \eta^{\sigma}(t) q^{\alpha}(t)|f(t)|^{1-\alpha},
\end{gathered}
$$

and $\delta_{1}$ is defined as in Theorem 3. Then (1) is oscillatory on $\left[t_{0}, \infty\right)_{\mathbb{T}}$.

Proof. Assume that $x$ is a nonoscillatory solution of (1) on $\left[t_{0}, \infty\right)_{\mathbb{T}}$. Without loss of generality, we may assume that there exists a $t_{1} \in\left[t_{0}, \infty\right)_{\mathbb{T}}$, such that $x(t)>0, x^{\sigma}(t)>0$ for all $t \in\left[t_{1}, \infty\right)_{\mathbb{T}}$. By assumption, we can choose $b_{1}>a_{1}>t_{1}$, then $q(t) \geq 0$ and $F\left(t, x^{\sigma}(t)\right) \leq 0$ on the interval $\left[a_{1}, b_{1}\right]_{\mathbb{T}}$. We define $\omega$ as in Theorem 3. Proceeding as in the proof of Theorem 3, we have (28). Hence, from (25), (28), and (47), we get

$$
\begin{aligned}
\omega^{\Delta}(t) \leq & -\frac{\eta^{\sigma}(t) p(t)}{r^{\sigma}(t)} \delta_{1}\left(\sigma(t), a_{1}\right)-\frac{\eta^{\sigma}(t) q(t)}{\left(x^{\sigma}(t)\right)^{1-\alpha}} \\
& -\eta^{\sigma}(t)|f(t)|\left(x^{\sigma}(t)\right)^{\alpha} \\
& -\frac{\eta^{\sigma}(t) x(t)}{\eta^{2}(t) r(t) x^{\sigma}(t)} \omega^{2}(t)+\eta^{\Delta}(t) \delta_{1}\left(t, a_{1}\right) .
\end{aligned}
$$

Set

$$
\begin{gathered}
G(x)=\frac{\eta^{\sigma}(t) q(t)}{x^{1-\alpha}}-\eta^{\sigma}(t)|f(t)| x^{\alpha}, \\
\lambda=\frac{1}{\alpha}, \quad \rho=\frac{1}{1-\alpha} .
\end{gathered}
$$

From Lemma 2, it is easy to see that

$$
G\left(x^{\sigma}\right) \geq \frac{1}{\alpha^{\alpha}(1-\alpha)^{1-\alpha}} \eta^{\sigma}(t) q^{\alpha}(t)|f(t)|^{1-\alpha}=\delta_{2}(t)
$$

Thus, combining (32), (50), and (52) and noticing that $\eta^{\Delta}(t) \geq$ 0 , we have

$$
\omega^{\Delta}(t) \leq-P_{1}\left(t, a_{1}\right)-\frac{1}{\eta(t) r(t)+\mu(t) \omega(t)} \omega^{2}(t),
$$

where $P_{1}$ is defined as in Theorem 7. Multiplying (53) by $\left(u^{\sigma}(t)\right)^{2}$ and integrating from $a_{1}$ to $b_{1}$, we get

$$
\begin{aligned}
\int_{a_{1}}^{b_{1}}\left(u^{\sigma}(t)\right)^{2} \omega^{\Delta}(t) \Delta t \leq & -\int_{a_{1}}^{b_{1}} P_{1}\left(t, a_{1}\right)\left(u^{\sigma}(t)\right)^{2} \Delta t \\
& -\int_{a_{1}}^{b_{1}} \frac{\left(u^{\sigma}(t)\right)^{2} \omega^{2}(t)}{\eta(t) r(t)+\mu(t) \omega(t)} \Delta t
\end{aligned}
$$

The rest of the argument proceeds as in Theorem 3 to get a contradiction to (47). The proof is complete.

Next, let us introduce the class of functions $Y$, which will be extensively used in the sequel.

Let $\mathbb{D}_{0}=\left\{(t, s) \in \mathbb{T}^{2}: t>s \geq t_{0}\right\}$ and $\mathbb{D}=\left\{(t, s) \in \mathbb{T}^{2}\right.$ : $\left.t \geq s \geq t_{0}\right\}$. We say that the function $H \in C_{\mathrm{rd}}(\mathbb{D}, \mathbb{R})$ belongs to the class $Y$, if

(i) $H(t, t)=0, t \geq t_{0}, H(t, s)>0$ on $\mathbb{D}_{0}$;

(ii) $H$ has continuous $\Delta$-partial derivatives $H^{\Delta_{t}}(t, s)$ and $H^{\Delta_{s}}(t, s)$ on $\mathbb{D}$ such that

$$
\begin{gathered}
H^{\Delta_{t}}(t, \sigma(s))=h_{1}(t, s) \sqrt{H(\sigma(t), \sigma(s))}, \\
H^{\Delta_{s}}(\sigma(t), s)=-h_{2}(t, s) \sqrt{H(\sigma(t), \sigma(s))},
\end{gathered}
$$

where $h_{1}$ and $h_{2} \in C_{\text {rd }}(\mathbb{D}, \mathbb{R})$.

Theorem 8. Assume that $\alpha>1$ and for any $T \in\left[t_{0}, \infty\right)_{\mathbb{T}}$, there exist constants $a_{k}$ and $b_{k} \in[T, \infty)_{\mathbb{T}}$, such that $a_{k}<b_{k}$, $k=1,2$, with

$$
\begin{gathered}
q(t) \geq 0, \quad \text { for } t \in\left[a_{1}, b_{1}\right]_{\mathbb{T}} \cup\left[a_{2}, b_{2}\right]_{\mathbb{T}}, \\
(-1)^{k} F\left(t, x^{\sigma}(t)\right) \geq(-1)^{k} f(t) \geq 0, \\
\text { for } t \in\left[a_{k}, b_{k}\right]_{\mathbb{T}}, \quad k=1,2,
\end{gathered}
$$


where $f \in C_{r d}\left(\left[t_{0}, \infty\right)_{\mathbb{T}}, \mathbb{R}\right)$. Furthermore, assume that there exists a function $\eta \in C_{r d}^{1}\left(\left[t_{0}, \infty\right)_{\mathbb{T}}, \mathbb{R}^{+}\right)$such that for some $H \in$ $Y$ and $c_{k} \in\left(a_{k}, b_{k}\right)_{\mathbb{T}}$,

$$
\begin{gathered}
\frac{1}{H\left(\sigma\left(c_{k}\right), \sigma\left(a_{k}\right)\right)} \int_{a_{k}}^{c_{k}}\left[H\left(\sigma(s), \sigma\left(a_{k}\right)\right) Q\left(s, a_{k}\right)\right. \\
\left.-\frac{\eta^{2}(s) r(s)}{4 \eta^{\sigma}(s) \delta\left(s, a_{k}\right)} \phi_{1}^{2}\left(s, a_{k}\right)\right] \Delta s \\
+\frac{1}{H\left(\sigma\left(b_{k}\right), \sigma\left(c_{k}\right)\right)} \int_{c_{k}}^{b_{k}}\left[H\left(\sigma\left(b_{k}\right), \sigma(s)\right) Q\left(s, a_{k}\right)\right. \\
\left.-\frac{\eta^{2}(s) r(s)}{4 \eta^{\sigma}(s) \delta\left(s, a_{k}\right)} \phi_{2}^{2}\left(b_{k}, s\right)\right]
\end{gathered}
$$$$
\times \Delta s>0, \quad k=1,2,
$$

where

$$
\begin{gathered}
\phi_{1}\left(s, a_{k}\right)=h_{1}\left(s, a_{k}\right)+\sqrt{H\left(\sigma(s), \sigma\left(a_{k}\right)\right)} \frac{\eta^{\Delta}(s)}{\eta(s)}, \\
\phi_{2}\left(b_{k}, s\right)=h_{2}\left(b_{k}, s\right)-\sqrt{H\left(\sigma\left(b_{k}\right), \sigma(s)\right)} \frac{\eta^{\Delta}(s)}{\eta(s)}, \\
Q\left(t, a_{k}\right)=\delta_{0}(t)+\frac{\eta^{\sigma}(t) p(t)}{r^{\sigma}(t)} \delta_{1}\left(\sigma(t), a_{k}\right), \\
\delta\left(t, a_{k}\right)=\int_{a_{k}}^{t} \frac{\Delta s}{r(s) e_{p / r^{\sigma}}\left(s, a_{k}\right)}\left(\int_{a_{k}}^{\sigma(t)} \frac{\Delta s}{r(s) e_{p / r^{\sigma}}\left(s, a_{k}\right)}\right)^{-1},
\end{gathered}
$$

and $\delta_{0}$ and $\delta_{1}$ are defined as in Theorem 3. Then (1) is oscillatory on $\left[t_{0}, \infty\right)_{\mathbb{T}}$.

Proof. Assume that $x$ is a nonoscillatory solution of (1) on $\left[t_{0}, \infty\right)_{\mathbb{T}}$. Without loss of generality, we may assume that there exists a $t_{1} \in\left[t_{0}, \infty\right)_{\mathbb{T}}$, such that $x(t)>0, x^{\sigma}(t)>0$ for all $t \in\left[t_{1}, \infty\right)_{\mathbb{T}}$. By assumption, we can choose $b_{1}>a_{1}>t_{1}$, then $q(t) \geq 0$ and $F\left(t, x^{\sigma}(t)\right) \leq 0$ on the interval $\left[a_{1}, b_{1}\right]_{\mathbb{T}}$. We define the function $\omega$ as in Theorem 3. Proceeding as in the proof of Theorem 3 and from (25) and (31), we get

$$
\omega^{\Delta}(t) \leq-Q\left(t, a_{1}\right)+\frac{\eta^{\Delta}(t)}{\eta(t)} \omega(t)-\frac{\eta^{\sigma}(t) x(t)}{\eta^{2}(t) r(t) x^{\sigma}(t)} \omega^{2}(t),
$$

where $Q$ is defined as in Theorem 7. Since $r(t) x^{\Delta}(t) e_{p / r^{\sigma}}\left(t, a_{1}\right)$ is nonincreasing on $\left[a_{1}, b_{1}\right]_{\mathbb{T}}$, we obtain

$$
\begin{aligned}
x^{\sigma}(t)-x(t) & =\int_{t}^{\sigma(t)} \frac{r(s) x^{\Delta}(s) e_{p / r^{\sigma}}\left(s, a_{1}\right)}{r(s) e_{p / r^{\sigma}}\left(s, a_{1}\right)} \Delta s \\
& \leq r(t) x^{\Delta}(t) e_{p / r^{\sigma}}\left(t, a_{1}\right) \int_{t}^{\sigma(t)} \frac{\Delta s}{r(s) e_{p / r^{\sigma}}\left(s, a_{1}\right)},
\end{aligned}
$$

hence

$$
\frac{x^{\sigma}(t)}{x(t)} \leq 1+\frac{r(t) x^{\Delta}(t) e_{p / r^{\sigma}}\left(t, a_{1}\right)}{x(t)} \int_{t}^{\sigma(t)} \frac{\Delta s}{r(s) e_{p / r^{\sigma}}\left(s, a_{1}\right)}
$$

From (25), we have

$$
\frac{r(t) x^{\Delta}(t) e_{p / r^{\sigma}}\left(t, a_{1}\right)}{x(t)}<\left(\int_{a_{1}}^{t} \frac{\Delta s}{r(s) e_{p / r^{\sigma}}\left(s, a_{1}\right)}\right)^{-1} .
$$

Therefore, from (61) and (62), we get

$$
\begin{aligned}
\frac{x^{\sigma}(t)}{x(t)} & <\int_{a_{1}}^{\sigma(t)} \frac{\Delta s}{r(s) e_{p / r^{\sigma}}\left(s, a_{1}\right)}\left(\int_{a_{1}}^{t} \frac{\Delta s}{r(s) e_{p / r^{\sigma}}\left(s, a_{1}\right)}\right)^{-1} \\
& =\frac{1}{\delta\left(t, a_{1}\right)} .
\end{aligned}
$$

Combining (59) and (63), we obtain

$$
\begin{aligned}
\omega^{\Delta}(t) \leq & -Q\left(t, a_{1}\right)+\frac{\eta^{\Delta}(t)}{\eta(t)} \omega(t) \\
& -\frac{\eta^{\sigma}(t) \delta\left(t, a_{1}\right)}{\eta^{2}(t) r(t)} \omega^{2}(t), \quad t \in\left[a_{1}, b_{1}\right]_{\mathbb{V}} .
\end{aligned}
$$

Multiplying both sides of (64) by $H(\sigma(s), \sigma(t))$ and integrating with respect to $s$ from $t$ to $c_{1}$ for $t \in\left(a_{1}, c_{1}\right]_{\mathbb{T}}$, we have

$$
\begin{aligned}
\int_{t}^{c_{1}} H(\sigma(s), \sigma(t)) Q\left(s, a_{1}\right) \Delta s \\
\leq-\int_{t}^{c_{1}} H(\sigma(s), \sigma(t)) \omega^{\Delta}(s) \Delta s \\
\quad+\int_{t}^{c_{1}} H(\sigma(s), \sigma(t)) \frac{\eta^{\Delta}(s)}{\eta(s)} \omega(s) \Delta s \\
\quad-\int_{t}^{c_{1}} H(\sigma(s), \sigma(t)) \frac{\eta^{\sigma}(s) \delta\left(s, a_{1}\right)}{\eta^{2}(s) r(s)} \omega^{2}(s) \Delta s .
\end{aligned}
$$

In view of (i) and (ii), we see that

$$
\begin{aligned}
\int_{t}^{c_{1}} H(\sigma(s), \sigma(t)) \omega^{\Delta}(s) \Delta s \\
=H\left(\sigma\left(c_{1}\right), \sigma(t)\right) \omega\left(c_{1}\right) \\
\quad-\int_{t}^{c_{1}} h_{1}(s, t) \sqrt{H(\sigma(s), \sigma(t))} \omega(s) \Delta s .
\end{aligned}
$$


Using (66) in (65) leads to

$$
\begin{aligned}
& \int_{t}^{c_{1}} H(\sigma(s), \sigma(t)) Q\left(s, a_{1}\right) \Delta s \\
& \leq-H\left(\sigma\left(c_{1}\right), \sigma(t)\right) \omega\left(c_{1}\right) \\
& -\int_{t}^{c_{1}} H(\sigma(s), \sigma(t)) \frac{\eta^{\sigma}(s) \delta\left(s, a_{1}\right)}{\eta^{2}(s) r(s)} \omega^{2}(s) \Delta s \\
& +\int_{t}^{c_{1}}\left(h_{1}(s, t) \sqrt{H(\sigma(s), \sigma(t))}\right. \\
& \left.+H(\sigma(s), \sigma(t)) \frac{\eta^{\Delta}(s)}{\eta(s)}\right) \omega(s) \Delta s \\
& =-H\left(\sigma\left(c_{1}\right), \sigma(t)\right) \omega\left(c_{1}\right) \\
& +\int_{t}^{c_{1}} \frac{\eta^{2}(s) r(s)}{4 \eta^{\sigma}(s) \delta\left(s, a_{1}\right)} \phi_{1}^{2}(s, t) \Delta s \\
& -\int_{t}^{c_{1}}\left(\sqrt{H(\sigma(s), \sigma(t)) \frac{\eta^{\sigma}(s) \delta\left(s, a_{1}\right)}{r(s)}} \frac{\omega(s)}{\eta(s)}\right. \\
& \left.-\frac{\eta(s) \sqrt{r(s)}}{2 \sqrt{\eta^{\sigma}(s) \delta\left(s, a_{1}\right)}} \phi_{1}(s, t)\right)^{2} \Delta s \\
& \leq-H\left(\sigma\left(c_{1}\right), \sigma(t)\right) \omega\left(c_{1}\right) \\
& +\int_{t}^{c_{1}} \frac{\eta^{2}(s) r(s)}{4 \eta^{\sigma}(s) \delta\left(s, a_{1}\right)} \phi_{1}^{2}(s, t) \Delta s .
\end{aligned}
$$

Letting $t \rightarrow a_{1}^{+}$in the above inequality, we get

$$
\begin{aligned}
& \frac{1}{H\left(\sigma\left(c_{1}\right), \sigma\left(a_{1}\right)\right)} \int_{a_{1}}^{c_{1}}\left[H\left(\sigma(s), \sigma\left(a_{1}\right)\right) Q\left(s, a_{1}\right)\right. \\
& \left.-\frac{\eta^{2}(s) r(s)}{4 \eta^{\sigma}(s) \delta\left(s, a_{1}\right)} \phi_{1}^{2}\left(s, a_{1}\right)\right] \Delta s \\
& \leq-\omega\left(c_{1}\right) .
\end{aligned}
$$

Similarly, multiplying both sides of (64) by $H(\sigma(t), \sigma(s))$ and integrating with respect to $s$ from $c_{1}$ to $t$ for $t \in\left[c_{1}, b_{1}\right)_{\mathbb{T}}$, we obtain

$$
\begin{aligned}
\int_{c_{1}}^{t} H(\sigma(t), \sigma(s)) Q\left(s, a_{1}\right) \Delta s \\
\leq-\int_{\mathcal{c}_{1}}^{t} H(\sigma(t), \sigma(s)) \omega^{\Delta}(s) \Delta s \\
\quad+\int_{c_{1}}^{t} H(\sigma(t), \sigma(s)) \frac{\eta^{\Delta}(s)}{\eta(s)} \omega(s) \Delta s
\end{aligned}
$$

$$
\begin{aligned}
& -\int_{c_{1}}^{t} H(\sigma(t), \sigma(s)) \frac{\eta^{\sigma}(s) \delta\left(s, a_{1}\right)}{\eta^{2}(s) r(s)} \omega^{2}(s) \Delta s \\
& \leq H\left(\sigma(t), \sigma\left(c_{1}\right)\right) \omega\left(c_{1}\right) \\
& -\int_{c_{1}}^{t} H(\sigma(t), \sigma(s)) \frac{\eta^{\sigma}(s) \delta\left(s, a_{1}\right)}{\eta^{2}(s) r(s)} \omega^{2}(s) \Delta s \\
& -\int_{c_{1}}^{t}\left(h_{2}(t, s) \sqrt{H(\sigma(t), \sigma(s))}\right. \\
& \left.-H(\sigma(t), \sigma(s)) \frac{\eta^{\Delta}(s)}{\eta(s)}\right) \omega(s) \Delta s \\
& =H\left(\sigma(t), \sigma\left(c_{1}\right)\right) \omega\left(c_{1}\right) \\
& +\int_{c_{1}}^{t} \frac{\eta^{2}(s) r(s)}{4 \eta^{\sigma}(s) \delta\left(s, a_{1}\right)} \phi_{2}^{2}(t, s) \Delta s \\
& -\int_{c_{1}}^{t}\left(\sqrt{H(\sigma(t), \sigma(s)) \frac{\eta^{\sigma}(s) \delta\left(s, a_{1}\right)}{r(s)}} \frac{\omega(s)}{\eta(s)}\right. \\
& \left.+\frac{\eta(s) \sqrt{r(s)}}{2 \sqrt{\eta^{\sigma}(s) \delta\left(s, a_{1}\right)}} \phi_{2}(t, s)\right)^{2} \Delta s \\
& \leq H\left(\sigma(t), \sigma\left(c_{1}\right)\right) \omega\left(c_{1}\right) \\
& +\int_{c_{1}}^{t} \frac{\eta^{2}(s) r(s)}{4 \eta^{\sigma}(s) \delta\left(s, a_{1}\right)} \phi_{2}^{2}(t, s) \Delta s .
\end{aligned}
$$

Letting $t \rightarrow b_{1}^{-}$in the above inequality, we get

$$
\begin{aligned}
& \frac{1}{H\left(\sigma\left(b_{1}\right), \sigma\left(c_{1}\right)\right)} \int_{c_{1}}^{b_{1}}\left[H\left(\sigma\left(b_{1}\right), \sigma(s)\right) Q\left(s, a_{1}\right)\right. \\
& \left.\quad-\frac{\eta^{2}(s) r(s)}{4 \eta^{\sigma}(s) \delta\left(s, a_{1}\right)} \phi_{2}^{2}\left(b_{1}, s\right)\right] \Delta s \\
& \leq \omega\left(c_{1}\right) .
\end{aligned}
$$

Adding (68) and (70), we get a contradiction to (57). This completes the proof.

Theorem 9. Assume that $\alpha<1$ and for any $T \in\left[t_{0}, \infty\right)_{\pi}$, there exist constants $a_{k}$ and $b_{k} \in[T, \infty)_{\mathbb{T}}$, such that $a_{k}<b_{k}$, $k=1,2$, with

$$
\begin{array}{r}
q(t) \geq 0 \quad \text { for } t \in\left[a_{1}, b_{1}\right]_{\mathbb{T}} \cup\left[a_{2}, b_{2}\right]_{\mathbb{T}}, \\
(-1)^{k} F\left(t, x^{\sigma}(t)\right) \geq(-1)^{k} f(t)\left(x^{\sigma}(t)\right)^{\alpha+1} \geq 0, \\
\text { for } t \in\left[a_{k}, b_{k}\right]_{\mathbb{T}}, k=1,2,
\end{array}
$$


where $f \in C_{r d}\left(\left[t_{0}, \infty\right)_{\mathbb{T}}, \mathbb{R}\right)$. Furthermore, assume that there exists a function $\eta \in C_{r d}^{1}\left(\left[t_{0}, \infty\right)_{\mathbb{T}}, \mathbb{R}^{+}\right)$such that for some $H \in$ $Y$ and $c_{k} \in\left(a_{k}, b_{k}\right)_{\mathbb{T}}$,

$$
\begin{gathered}
\frac{1}{H\left(\sigma\left(c_{k}\right), \sigma\left(a_{k}\right)\right)} \int_{a_{k}}^{c_{k}}\left[H\left(\sigma(s), \sigma\left(a_{k}\right)\right) \widetilde{Q}\left(s, a_{k}\right)\right. \\
\left.-\frac{\eta^{2}(s) r(s)}{4 \eta^{\sigma}(s) \delta\left(s, a_{k}\right)} \phi_{1}^{2}\left(s, a_{k}\right)\right] \Delta s \\
+\frac{1}{H\left(\sigma\left(b_{k}\right), \sigma\left(c_{k}\right)\right)} \int_{c_{k}}^{b_{k}}\left[H\left(\sigma\left(b_{k}\right), \sigma(s)\right) \widetilde{Q}\left(s, a_{k}\right)\right. \\
\left.-\frac{\eta^{2}(s) r(s)}{4 \eta^{\sigma}(s) \delta\left(s, a_{k}\right)} \phi_{2}^{2}\left(b_{k}, s\right)\right]
\end{gathered}
$$$$
\times \Delta s>0, \quad k=1,2,
$$

where

$$
\widetilde{Q}\left(t, a_{k}\right)=\delta_{2}(t)+\frac{\eta^{\sigma}(t) p(t)}{r^{\sigma}(t)} \delta_{1}\left(\sigma(t), a_{k}\right) .
$$

$\delta_{1}$ is defined as in Theorem $3, \delta_{2}$ is defined as in Theorem 7 , and $\phi_{1}, \phi_{2}$, and $\delta$ are defined as in Theorem 8. Then (1) is oscillatory on $\left[t_{0}, \infty\right)_{\mathbb{T}}$.

The proof of Theorem 9 is similar to that of Theorem 8 , so we omit the proof.

Remark 10. The main results in this paper can also be extended to the following second order damped dynamic equations with mixed nonlinearities:

$$
\begin{aligned}
(r(t) & \left.x^{\Delta}(t)\right)^{\Delta}+p(t) x^{\Delta \sigma}(t)+q_{0}(t) x\left(\tau_{0}(t)\right) \\
& +q_{1}(t)\left|x\left(\tau_{1}(t)\right)\right|^{\beta-1} x\left(\tau_{1}(t)\right) \\
& +q_{2}(t)\left|x\left(\tau_{2}(t)\right)\right|^{\gamma-1} x\left(\tau_{2}(t)\right)=F\left(t, x^{\sigma}(t)\right),
\end{aligned}
$$

where $\gamma>1>\beta>0, \tau_{i}(t) \leq \sigma(t), i=0,1,2$, or the more general equation

$$
\begin{aligned}
(r(t) & \left.x^{\Delta}(t)\right)^{\Delta}+p(t) x^{\Delta \sigma}(t) \\
& +\sum_{i=0}^{n} q_{i}(t)\left|x\left(\tau_{i}(t)\right)\right|^{\alpha_{i}-1} x\left(\tau_{i}(t)\right)=F\left(t, x^{\sigma}(t)\right)
\end{aligned}
$$

on any arbitrary time scale $\mathbb{T}$, where $\alpha_{0}=1, \alpha_{1}>\alpha_{2}>\cdots>$ $\alpha_{m}>1>\alpha_{m+1}>\cdots>\alpha_{n}>0$ and $\tau_{i}$ are nondecreasing rdcontinuous functions on $\mathbb{R}$ with $\tau_{i}(t) \leq \sigma(t), i=0,1, \ldots, n$. Due to the limited space, we omit it here and leave it to the readers who are interested in this problem.

\section{Examples}

In this section, we will show the applications of our interval oscillation criteria in two examples. Firstly, we will give an example to illustrate Theorem 3.
Example 1. Consider the following second order forced difference equations with damping:

$$
\begin{aligned}
& \Delta\left(t\left(\sin \frac{\pi t}{4}+2\right) \Delta x(t)\right) \\
& \quad-\frac{t^{2}-1}{t^{2}}\left(\sin \frac{\pi(t+1)}{4}+2\right) \Delta x(t) \\
& \quad+\frac{c_{0}}{(t+1)^{2}}\left(\sin \frac{\pi t}{4}+2\right) x^{2}(t)=-\cos \frac{\pi t}{4},
\end{aligned}
$$

for $t \geq 2$, where $c_{0}$ is a positive constant. Here

$$
\begin{gathered}
r(t)=t\left(\sin \frac{\pi t}{4}+2\right), \\
p(t)=-\frac{t^{2}-1}{t^{2}}\left(\sin \frac{\pi(t+1)}{4}+2\right), \\
q(t)=\frac{c_{0}}{(t+1)^{2}}\left(\sin \frac{\pi t}{4}+2\right), \\
F(t, x(t))=f(t)=-\cos \frac{\pi t}{4}, \quad \alpha=2 .
\end{gathered}
$$

Let

$$
\begin{gathered}
a_{1}=8 h, \quad b_{1}=a_{2}=8 h+2, \\
b_{2}=8 h+4, \quad h=1,2, \ldots,
\end{gathered}
$$

such that

$$
\begin{gathered}
q(t) \geq 0, \quad(-1)^{k} f(t) \geq 0, \\
t \in[8 h, 8 h+2) \cup[8 h+2,8 h+4), \quad k=1,2 .
\end{gathered}
$$

For $t \geq 2$, we obtain

$$
\begin{aligned}
\delta_{1}\left(\sigma(t), a_{k}\right) & \\
= & \frac{1}{\left(1+\mu(t)\left(p(t) / r^{\sigma}(t)\right)\right) e_{p / r^{\sigma}}\left(t, a_{k}\right)} \\
& \times\left(\int_{a_{k}}^{\sigma(t)} \frac{1}{\left(1+\mu(s)\left(p(s) / r^{\sigma}(s)\right)\right) r(s) e_{p / r^{\sigma}}\left(s, a_{k}\right)} \Delta s\right)^{-1} \\
\leq & \frac{t}{t-1} \delta_{1}\left(t, a_{k}\right) .
\end{aligned}
$$


Setting $\eta(t)=1 / t$ and $u(t)=\sin (\pi t / 2)$, we have

$$
\begin{aligned}
P\left(t, a_{k}\right) \geq & 2(t+1)\left(\frac{c_{0}}{(t+1)^{2}}\left(\sin \frac{\pi t}{4}+2\right)\right)^{1 / 2}\left|-\cos \frac{\pi t}{4}\right|^{1 / 2} \\
& =2\left(c_{0}\left(\sin \frac{\pi t}{4}+2\right)\left|\cos \frac{\pi t}{4}\right|\right)^{1 / 2}, \\
\sum_{j=a_{1}}^{b_{1}-1}\left(\eta(j) r(j)(\Delta u(j))^{2}-P\left(j, a_{1}\right) u^{2}(j+1)\right) & \\
\leq & \sum_{j=8 h}^{8 h+1}\left(\frac{1}{j} \cdot j\left(\sin \frac{\pi j}{4}+2\right)\left(\sin \frac{\pi(j+1)}{2}-\sin \frac{\pi j}{2}\right)^{2}\right. \\
& \left.-2\left(c_{0}\left(\sin \frac{\pi j}{4}+2\right) \cos \frac{\pi j}{4}\right)^{1 / 2} \sin ^{2} \frac{\pi(j+1)}{2}\right)^{2} \\
= & \frac{\sqrt{2}}{2}+4-2\left(2 c_{0}\right)^{1 / 2} \cdot
\end{aligned}
$$

Then by Theorem 3, every solution of (76) is oscillatory if

$$
c_{0} \geq \frac{1}{2}\left(\frac{\sqrt{2}}{4}+2\right)^{2}
$$

Next, we will give an example to illustrate Theorem 7.

Example 2. Consider the following second order forced differential equations with damping:

$$
\begin{aligned}
& \left(t(\sin 2 t+2) x^{\prime}(t)\right)^{\prime}-(\sin 2 t+2) x^{\prime}(t)+\frac{c_{0} \cos ^{2} 2 t}{t^{1 / \alpha}} x^{\alpha}(t) \\
& =-\sin 2 t, \quad t \geq 1,
\end{aligned}
$$

where $c_{0}$ is a positive constant. Here,

$$
\begin{array}{r}
r(t)=t(\sin 2 t+2), \quad p(t)=-\sin 2 t-2, \\
q(t)=\frac{c_{0} \cos ^{2} 2 t}{t^{1 / \alpha}}, \quad F(t, x(t))=f(t)=-\sin 2 t, \\
\quad \alpha<1 .
\end{array}
$$

Let

$$
\begin{gathered}
a_{1}=2 h \pi, \quad b_{1}=a_{2}=2 h \pi+\frac{\pi}{2}, \\
b_{2}=2 h \pi+\pi, \quad h=1,2, \ldots,
\end{gathered}
$$

such that

$$
\begin{gathered}
q(t) \geq 0, \quad(-1)^{k} f(t) \geq 0, \\
t \in\left[2 h \pi, 2 h \pi+\frac{\pi}{2}\right) \cup\left[2 h \pi+\frac{\pi}{2}, 2 h \pi+\pi\right), \quad k=1,2 .
\end{gathered}
$$

Setting $\eta(t)=1 / t$ and $u(t)=\sin 2 t$, we obtain

$$
\begin{aligned}
& P_{1}\left(t, a_{k}\right)= \frac{t}{\alpha^{\alpha}(1-\alpha)^{1-\alpha}}\left(\frac{c_{0} \cos ^{2} 2 t}{t^{1 / \alpha}}\right)^{\alpha}|-\sin 2 t|^{1-\alpha} \\
&= \frac{c_{0}^{\alpha}}{\alpha^{\alpha}(1-\alpha)^{1-\alpha}} \cos ^{2 \alpha} 2 t|-\sin 2 t|^{1-\alpha}, \\
& \int_{a_{1}}^{b_{1}}\left(\eta(t) r(t)\left(u^{\prime}(t)\right)^{2}-P_{1}\left(t, a_{1}\right) u^{2}(t)\right) \mathrm{d} t \\
&=\int_{0}^{\pi / 2}\left(\frac{1}{t} \cdot t(\sin 2 t+2)(2 \cos 2 t)^{2}\right. \\
&\left.\quad=\frac{6}{5} \sqrt{\pi}+\pi-\frac{c_{0}^{\alpha}}{4 \alpha^{\alpha}(1-\alpha)^{1-\alpha}} \cos ^{2 \alpha} 2 t \sin ^{3-\alpha} 2 t\right) \mathrm{d} t \\
& \quad \times \frac{\Gamma(2-\alpha / 2) \Gamma(\alpha+1 / 2)}{\Gamma((\alpha+5) / 2)}
\end{aligned}
$$

where $\Gamma$ is the gamma function. Then by Theorem 7 , every solution of (83) is oscillatory if

$$
\frac{6}{5} \sqrt{\pi}+\pi \leq \frac{c_{0}^{\alpha}}{4 \alpha^{\alpha}(1-\alpha)^{1-\alpha}} \frac{\Gamma(2-\alpha / 2) \Gamma(\alpha+1 / 2)}{\Gamma((\alpha+5) / 2)}
$$

\section{Acknowledgments}

The authors sincerely thank the reviewers for their valuable suggestions and useful comments that have led to the present improved version of the original paper. This paper is supported by the Natural Science Foundation of China (11071143, 60904024, and 11026112), Natural Science Outstanding Youth Foundation of Shandong Province (JQ201119), Shandong Provincial Natural Science Foundation (ZR2012AM009, ZR2010AL002, and ZR2011AL007), and also by Natural Science Foundation of Educational Department of Shandong Province (J11LA01).

\section{References}

[1] S. Hilger, "Analysis on measure chains-a unified approach to continuous and discrete calculus," Results in Mathematics. Resultate der Mathematik, vol. 18, no. 1-2, pp. 18-56, 1990.

[2] M. Bohner and A. Peterson, Dynamic Equations on Time Scales, An Introduction with Applications, Birkhäuser, Boston, Mass, USA, 2001.

[3] M. Bohner and A. Peterson, Advances in Dynamic Equations on Time Scales, Birkhäuser, Boston, Mass, USA, 2003.

[4] W.-T. Li, "Interval oscillation criteria for second-order quasilinear nonhomogeneous differential equations with damping," Applied Mathematics and Computation, vol. 147, no. 3, pp. 753763, 2004.

[5] E. M. Elabbasy and T. S. Hassan, "Interval oscillation for second order sublinear differential equations with a damping term," 
International Journal of Dynamical Systems and Differential Equations, vol. 1, no. 4, pp. 291-299, 2008.

[6] Y. G. Sun and J. S. W. Wong, "Oscillation criteria for second order forced ordinary differential equations with mixed nonlinearities," Journal of Mathematical Analysis and Applications, vol. 334, no. 1, pp. 549-560, 2007.

[7] Y. G. Sun and F. W. Meng, "Interval criteria for oscillation of second-order differential equations with mixed nonlinearities," Applied Mathematics and Computation, vol. 198, no. 1, pp. 375381, 2008.

[8] Y. Huang and F. Meng, "Oscillation criteria for forced secondorder nonlinear differential equations with damping," Journal of Computational and Applied Mathematics, vol. 224, no. 1, pp. 339-345, 2009.

[9] T. S. Hassan, L. Erbe, and A. Peterson, "Forced oscillation of second order differential equations with mixed nonlinearities," Acta Mathematica Scientia B, vol. 31, no. 2, pp. 613-626, 2011.

[10] S. H. Saker, R. P. Agarwal, and D. O'Regan, "Oscillation of second-order damped dynamic equations on time scales," Journal of Mathematical Analysis and Applications, vol. 330, no. 2, pp. 1317-1337, 2007.

[11] L. Erbe, T. S. Hassan, and A. Peterson, "Oscillation criteria for nonlinear damped dynamic equations on time scales," Applied Mathematics and Computation, vol. 203, no. 1, pp. 343-357, 2008.

[12] W. Chen, Z. Han, S. Sun, and T. Li, "Oscillation behavior of a class of second-order dynamic equations with damping on time scales," Discrete Dynamics in Nature and Society, vol. 2010, Article ID 907130, 15 pages, 2010.

[13] T. S. Hassan, L. Erbe, and A. Peterson, "Oscillation of second order superlinear dynamic equations with damping on time scales," Computers \& Mathematics with Applications, vol. 59, no. 1, pp. 550-558, 2010.

[14] S. R. Grace, R. P. Agarwal, B. Kaymakçalan, and W. Saejie, "Oscillation theorems for second order nonlinear dynamic equations," Journal of Applied Mathematics and Computing, vol. 32, no. 1, pp. 205-218, 2010.

[15] T. S. Hassan, "Oscillation criteria for half-linear dynamic equations on time scales," Journal of Mathematical Analysis and Applications, vol. 345, no. 1, pp. 176-185, 2008.

[16] Y. Sahiner, "Oscillation of second-order delay differential equations on time scales," Nonlinear Analysis: Theory, Methods \& Applications, vol. 63, pp. 1073-1080, 2005.

[17] S. Sun, Z. Han, and C. Zhang, "Oscillation of second-order delay dynamic equations on time scales," Journal of Applied Mathematics and Computing, vol. 30, no. 1-2, pp. 459-468, 2009.

[18] S. Sun, Z. Han, P. Zhao, and C. Zhang, "Oscillation for a class of second-order Emden-Fowler delay dynamic equations on time scales," Advances in Difference Equations, vol. 2010, Article ID 642356, 15 pages, 2010.

[19] Z. Han, S. Sun, T. Li, and C. Zhang, "Oscillatory behavior of quasilinear neutral delay dynamic equations on time scales," Advances in Difference Equations, vol. 2010, Article ID 450264, 24 pages, 2010.

[20] Z. Han, S. Sun, and B. Shi, "Oscillation criteria for a class of second-order Emden-Fowler delay dynamic equations on time scales," Journal of Mathematical Analysis and Applications, vol. 334, no. 2, pp. 847-858, 2007.

[21] Z. Han, T. Li, S. Sun, and C. Zhang, "Oscillation for secondorder nonlinear delay dynamic equations on time scales," Advances in Difference Equations, vol. 2009, Article ID 756171, 13 pages, 2009.
[22] Y. Sun, Z. Han, T. Li, and G. Zhang, "Oscillation criteria for second-order quasilinear neutral delay dynamic equations on time scales," Advances in Difference Equations, vol. 2010, Article ID 512437, 14 pages, 2010.

[23] Y. Sun, Z. Han, and T. Li, "Oscillation criteria for second-order quasilinear neutral dynamic equations," Journal of University of Jinan, vol. 24, pp. 308-311, 2010.

[24] Z. Han, T. Li, S. Sun, and F. Cao, "Oscillation criteria for third order nonlinear delay dynamic equations on time scales," Annales Polonici Mathematici, vol. 99, no. 2, pp. 143-156, 2010.

[25] Z. Han, T. Li, S. Sun, and C. Zhang, "Oscillation behavior of third-order neutral Emden-Fowler delay dynamic equations on time scales," Advances in Difference Equations, vol. 2010, Article ID 586312, 23 pages, 2010.

[26] L. Erbe, A. Peterson, and S. H. Saker, "Oscillation criteria for a forced second-order nonlinear dynamic equation," Journal of Difference Equations and Applications, vol. 14, no. 10-11, pp. 9971009, 2008.

[27] D. R. Anderson, "Interval criteria for oscillation of nonlinear second-order dynamic equations on time scales," Nonlinear Analysis. Theory, Methods \& Applications, vol. 69, no. 12, pp. 4614-4623, 2008.

[28] D. R. Anderson, "Oscillation of second-order forced functional dynamic equations with oscillatory potentials," Journal of Difference Equations and Applications, vol. 13, no. 5, pp. 407-421, 2007.

[29] M. Bohner and C. C. Tisdell, "Oscillation and nonoscillation of forced second order dynamic equations," Pacific Journal of Mathematics, vol. 230, no. 1, pp. 59-71, 2007.

[30] L. Erbe, T. S. Hassan, A. Peterson, and S. H. Saker, "Interval oscillation criteria for forced second-order nonlinear delay dynamic equations with oscillatory potential," Dynamics of Continuous, Discrete \& Impulsive Systems A, vol. 17, no. 4, pp. 533-542, 2010.

[31] T. Li, Z. Han, S. Sun, and C. Zhang, "Forced oscillation of second-order nonlinear dynamic equations on time scales," Electronic Journal of Qualitative Theory of Differential Equations, vol. 60, pp. 1-8, 2009.

[32] L. Erbe, T. Hassan, and A. Peterson, "Oscillation criteria for forced second-order functional dynamic equations with mixed nonlinearities on time scales," Advances in Dynamical Systems and Applications, vol. 5, no. 1, pp. 61-73, 2010.

[33] R. P. Agarwal and A. Zafer, "Oscillation criteria for secondorder forced dynamic equations with mixed nonlinearities," Advances in Difference Equations, vol. 2009, Article ID 938706, 20 pages, 2009.

[34] Y. Sun, "Forced oscillation of second-order superlinear dynamic equations on time scales," Electronic Journal of Qualitative Theory of Differential Equations, vol. 44, pp. 1-11, 2011.

[35] D. R. Anderson and S. H. Saker, "Interval oscillation criteria for forced Emden-Fowler functional dynamic equations with oscillatory potential," Science China Mathematics, vol. 55, 2012.

[36] E. F. Beckenbach and R. Bellman, Inequalities, Second Revised Printing, Springer, New York, NY, USA, 1965. 


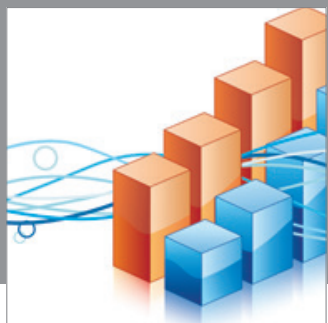

Advances in

Operations Research

mansans

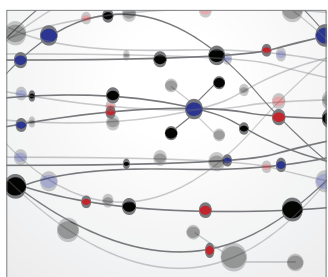

The Scientific World Journal
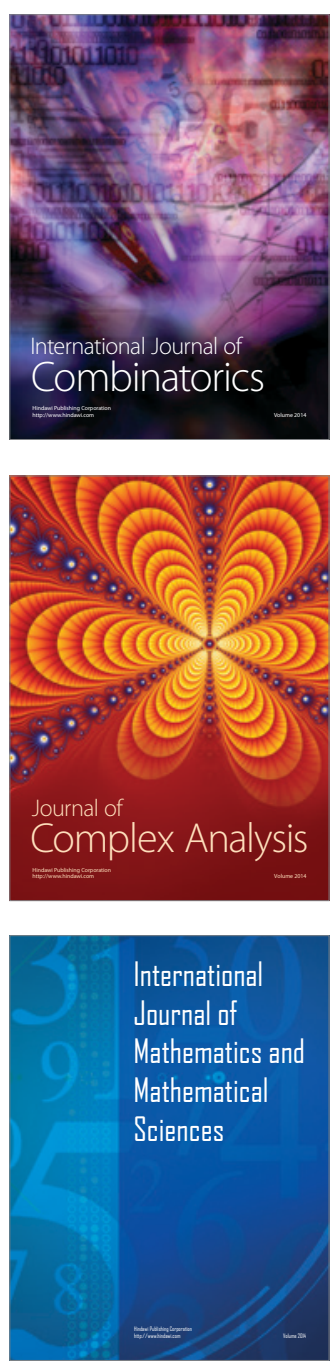
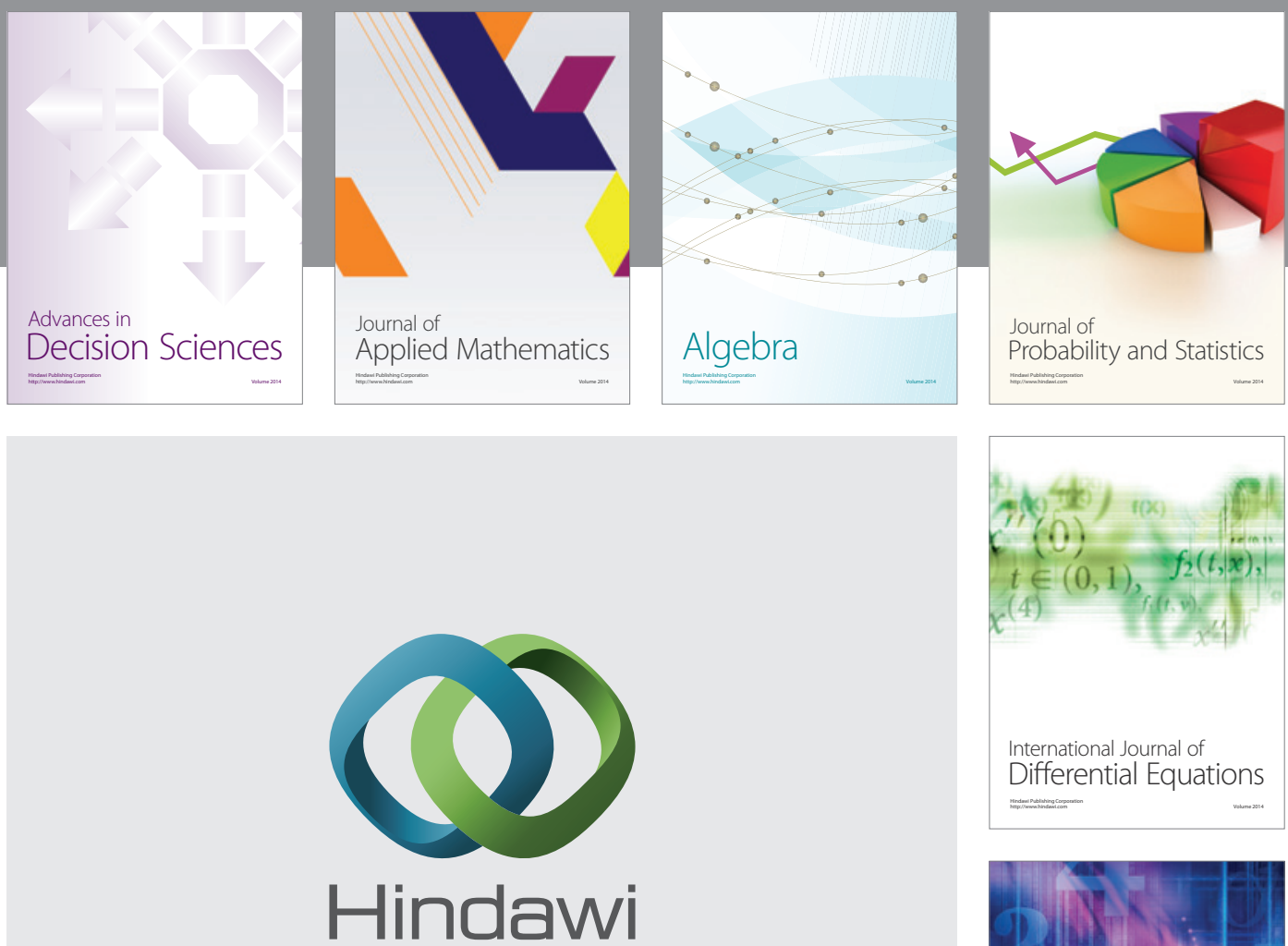

Submit your manuscripts at http://www.hindawi.com
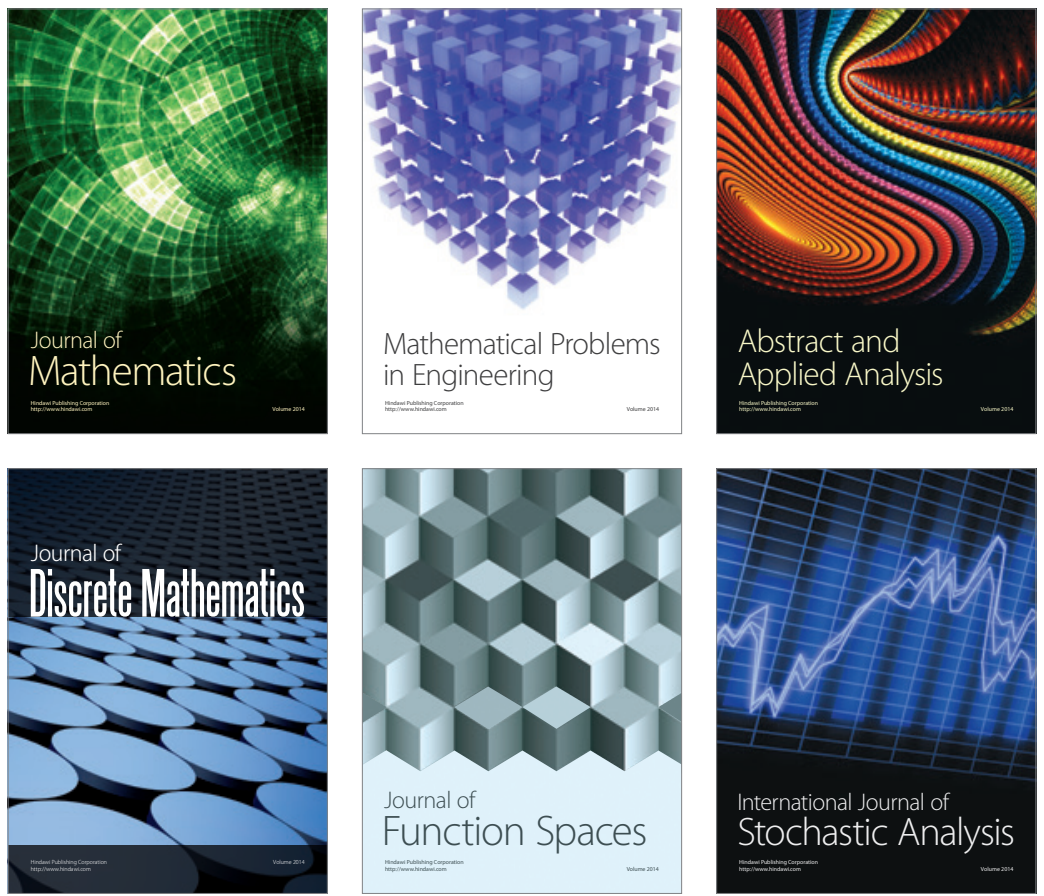

Journal of

Function Spaces

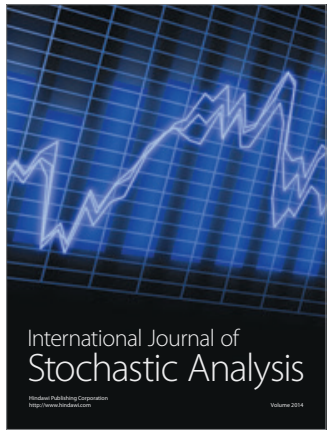

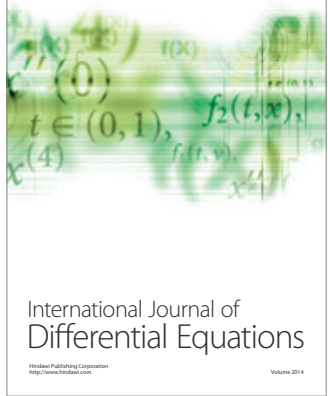
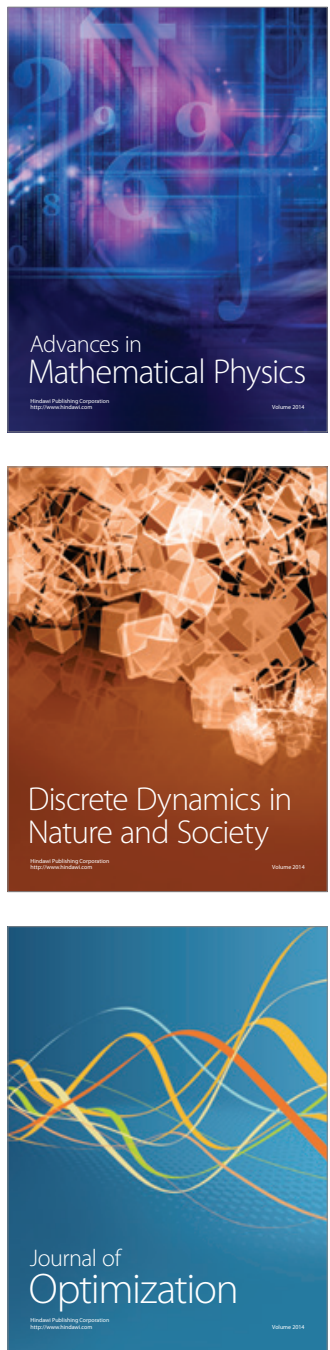